\title{
APPLICATION OF THE MATHEMATICAL METHODS OF THE BRAID GROUP THEORY TO QUANTIZATION OF MANY-PARTICLE SYSTEMS
}

\author{
D. J $\mathrm{ACAK}$ \\ Institute of Mathematics, Technical University of Wrocław \\ Wybrzeże Wyspiańskiego 27, 50-370 Wrocław, Poland \\ L. JACAK AND K. WIECZOREK \\ Institute of Physics, Technical University of Wroclaw \\ Wybrzeże Wyspiańskiego 27, 50-370 Wroclaw, Poland
}

(Received June 7, 1993)

\begin{abstract}
The problem of the non-standard statistics for one-, two- and three-dimensional systems of $N$ identical particles on various manifolds is reviewed in terms of the braid group theory. The braid groups together with their unitary representations are studied for the line, circle, plane, sphere, torus and the three-dimensional Euclidean space. Nonequivalent quantizations of several physical systems are presented.
\end{abstract}

PACS numbers: 03.65.Bz, 05.30.-d

\section{Introduction}

The topology of the configuration space for many-particle systems has important consequences in physics. This has been perceived at the end of sixties (after description of the magnetic monopole by Polyakov and 't Hooft) - cf. e.g. Schulman, Laidlaw and De Witt $[1,2]$. They studied three-dimensional systems and discovered that quantum statistics has the topological origin. Leinaas and Myrheim (cf. [3]) returned to this problem in 1977. They considered one-, two- and three-dimensional systems and indicated that one- and two-dimensional quantum mechanics is more complicated than its three-dimensional version. They found possibilities of existing new and exotic (nonboson and nonfermion) statistics. The two-dimensional case was clearly connected with a topological structure of the configuration space for a planar system. Recently, these problems focus once more the interest in physics of condensed matter. It is connected with two important 
discoveries: the fractional quantum Hall effect [4] and high-temperature superconductivity [5]. In both cases physical systems can be regarded as two-dimensional (a sharp quantum well for the fractional quantum Hall effect and a superconducting $\mathrm{Cu}-\mathrm{O}$ plane for high-temperature superconductors). The both phenomena have not been explained by standard three-dimensional physics and need new idea of current constructions for two-dimensional quantum physics. On the other hand, some models proposed by Wilczek in 1982 (cf. $[6,7]$ ) supply background for understanding the physics of two-dimensional quantum systems. Wilczek showed that quantum particles in two dimensions behave as objects with magnetic flux associated (the complex flux-particle was called by Wilczek as anyon). This subject was further considered and at the end of eighties it led to an exotic concept of the anyon superconductivity [8].

In the present paper we describe the mathematical background for all those phenomena using the uniform formalism for various $1 \mathrm{D}, 2 \mathrm{D}$ and $3 \mathrm{D}$ physical systems.

\section{The configuration space}

So far, there has been no way of direct inclusion of topology into quantum-mechanical description. The topology effects are, however, well understood on the classical level, therefore, one studies topology of the classical configuration space and transforms topological results onto quantum mechanics via quantization procedure.

The construction of a configuration space is the standard procedure (cf. [1, 2]). Let us consider $N$-particle system on the manifold $M$. Each point of the configuration space describes one of the possible configurations of the system. For distinguishable particles it is the Cartesian product $M^{N}$. Indistinguishability of identical particles leads to identifying the points of $M^{N}$ which differ only by a permutation of the particle indices. It means that $M^{N}$ is replaced by the so-called orbit space $M^{N} / S_{N}$, where $S_{N}$ is the permutation group of $N$ elements ("/" denotes the quotient structure). The action of an element of $S_{N}$ on $M^{N}$ describes a class of abstraction of the orbit space. There are, however, some problems with singular points in $M^{N} / S_{N}$ when two or more particle coordinates coincide (they are fixed points of $S_{N}$ action on $M^{N}$ ): It is not quite evident how to perform quantization of a system together with the configuration space with these singular points (the standard procedure leads to an unphysical result - only theories with Bose-Einstein statistics, cf. [3]). This problem could be removed if all singular points are excluded. These singular points are also called diagonal points and their set is denoted by $\Delta$. Hence, the configuration space suitable for quantization attains the form

$$
Q_{N}(M)=\left(M^{N} \backslash \Delta\right) / S_{N} .
$$

Moreover, let us define the configuration space for distinguishable particles in the following form:

$$
F_{N}(M)=M^{N} \backslash \Delta .
$$

This last notation is artificial from the physical point of view - it will be, however, useful in further considerations. 
The connection between $F_{N}(M)$ and $Q_{N}(M)$ can be described as a fibration $F_{N}(M)$ over the space $Q_{N}(M)$ with epimorphism $h_{S_{N}}$ which is connected with action of $S_{N}$ on $F_{N}(M)$. Because each fibre $\left[F_{N}(M)\right]_{x}, x \in Q_{N}(M)$ is a discrete space (it has $N$ ! elements) and fibration $h_{S_{N}}$ is locally trivial then $F_{N}(M)$ is the covering space for $Q_{N}(M)$. In other words, exclusion of diagonal points $\Delta$ from $F_{N}(M)$ allows freely acting of $S_{N}$ in $F_{N}(M)$ and homomorphism from $F_{N}(M)$ to orbit space $Q_{N}(M)$ is a covering projection (cf. [8]).

The configuration space $Q_{N}(M)$ requires a special kind of symmetry of the system. In three-dimensional physics transformations connected with this symmetry were understood as interchange of the wave function arguments. Leinaas and Myrheim in their paper [3] noticed that it is not a physical operation and it leads even to a paradox. If the distance between two particles is large (e.g., in a cosmic scale) they should rather be considered as distinguishable. In order to omit such a complication Leinaas and Myrheim proposed to understand the particle interchanging intimately connected with the way of its realization. It leads to the concept of symmetry connected rather with transport of the system along a closed path in $Q_{N}(M)$. It can further be expressed in terms of Feynman's path-integral formalism. When the space of all trajectories of the system is continuous then addition (in path-integral construction) of a particular trajectory resolves itself to weighing it in the classical limit. For classical equation of motion the appropriate weights are determined by varying the action with respect of infinitesimally nearby paths. They cannot, however, guide a choice of weights for trajectories which cannot be connected by a continuous deformation. But for some $Q_{N}(M)$ spaces the trajectories fall into disconnected pieces. The weights of partial amplitudes are argued to be referred rather to classes of homotopy of trajectories than to particular trajectories. Laidlaw and De Witt proved (cf. [2]) that these weights are determined by unitary representations of the fundamental group (i.e. the first homotopy group) of the configuration space.

Note that the first homotopy group is the group of closed paths and hence a construction is needed which allows one to describe also open trajectories. For the paths with the initial point $x_{1}$ and the final point $x_{2}$ one can construct an artificial trajectory from $x_{2}$ to $x_{1}$ which closes the open paths. If, however, $x_{1}=x_{2}$ then the trajectories which link these points describe an interchange of particles and hence the fundamental group refers to the statistical symmetry of identical particle system. Elements of the first homotopy group represent all possible nonequivalent replacements of particles. Hence, the statistical properties of the wave functions are described by unitary representations of the first homotopy group. More accurately, when $x$ is taken along a closed loop in $Q_{N}(M)$ then $\Psi(x)$ must transform according to unitary representation of the first homotopy group for $Q_{N}(M)$. Since the representation of $\pi_{1}\left(Q_{N}(M)\right)$ in general is nonunique, various quantum theories are possible for the same classical system. It holds both for one-dimensional unitary representations of $\pi_{1}$ and multi-valued irreducible unitary representations of $\pi_{1}$. For 3D manifolds two various one-dimensional representations of relevant homotopy group correspond to bosons and fermions while multivalued representations of the same group describe the so-called parastatistics 3D theories. In 2D case (and 1D) the collection of quantum theories is much more rich and corresponds to 
a quite other structure of the appropriate fundamental group: It will be discussed in the following chapters.

\section{Braid group}

The fundamental group of a configuration space is often called a braid group. For various manifolds $M$ it is rather a well-described mathematical object, (cf. [9]).

Let us consider $N$-particle system on the manifold $M$. The full braid group (or simply - the braid group) of $M$ is, by definition, the fundamental group $\pi_{1}(A)$, where $A$ is the configuration space: $A=Q_{N}(M)=\left(M^{N} \backslash \Delta\right) / S_{N}$. The pure braid group (or the unpermuted braid group) is the fundamental group $\pi_{1}(B)$, where $B=F_{N}(M)=M^{N} \backslash \Delta$. An element of $\pi_{1}\left(F_{N}(M)\right)$ can be considered as a homotopy class of paths in the space $M^{N} \backslash \Delta$, whose (fixed) initial and final points are the same. Let us denote the collection of generators of $\pi_{1}\left(F_{N}(M)\right)$ by $\mathcal{L}$. The set $\mathcal{L}$ contains all classes of noncontractable closed paths for which all particles except one stay in their initial positions. This one particle describes its own noncontractable closed curve on the manifold $M$. The origin of this noncontractability could be other particles or the peculiar topology of the manifold $M$ (e.g., hole, topological defect). An element of the group $\pi_{1}\left(Q_{N}(M)\right)$ can be regarded as a homotopy class of paths in $M^{N} \backslash \Delta$, whose initial and final points are related by a permutation of particle coordinates or, on the other hand, it is a homotopy class of closed paths in $Q_{N}(M)$ with fixed initial points. Hence, in general $\pi_{1}\left(Q_{N}(M)\right)$ has two sets of generators: $\mathcal{L}$ and $\mathcal{P}$. The set $\mathcal{P}$ contains the homotopy classes of closed paths which describe elementary operations of interchange of two neighbouring patricles. These operations are denoted by $\sigma_{i}, 1 \leq i \leq N-1$. The generator $\sigma_{i}$ is the class of closed paths in $Q_{N}(M)$ which interchange the coordinates of particles with $i$ and $i+1$ indices without creating any noncontractable loop and without moving any other particle. The group generated by elements of $\mathcal{P}$ is denoted by $\Sigma_{N}(M)$. It is obvious that if $M$ is simply connected then

$$
\pi_{1}\left(Q_{N}(M)\right)=\Sigma_{N}(M)
$$

and $\pi_{1}\left(F_{N}(M)\right)$ is a subgroup of $\Sigma_{N}(M)$. If $M$ is not simply connected then the sets $\mathcal{L}$ and $\mathcal{P}$ are separated. In this case relations between generators from the sets $\mathcal{L}$ and $\mathcal{P}$ can have very complicated structure and their form depends on $M$ (they are especially nontrivial when $\operatorname{dim} M=2)$. It is easy to see also that $\pi_{1}\left(F_{N}(M)\right)$ is a normal subgroup of $\pi_{1}\left(Q_{N}(M)\right)$ for any $M$.

\section{Braid group for the Euclidean plane $R^{2}$}

From the physical point of view the braid groups for two-dimensional manifolds are a very interesting case. The most important example is the braid group of the Euclidean plane $R^{2}$. This braid group was described first by Artin and $\pi_{1}\left(Q_{N}\left(R^{2}\right)\right)$ is called Artin classical braid group and it is denoted by $B_{N}$ (cf. [10]). It has a very simple graphical illustration (the so-called geometric braid), cf. Fig. 1 . On the two horizontial lines (in Fig. 1) configurations of the system are presented. These two lines are joined by $N$ strings. Each string represents the path of one 


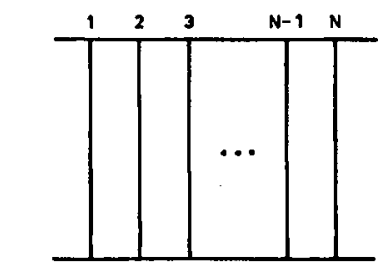

(a)

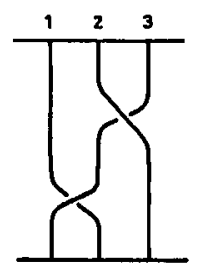

(b)

Fig. 1. Examples of the geometric braids: a) the trivial $N$-braid, b) the nontrivial 3-braid.

particle in the real space. The set of $N$ such strings represents a homotopy class of closed paths in the configuration space $Q_{N}(M)$, i.e. an element of $B_{N}$. Strings cannot cross because of excluding diagonal points (collected in the set $\Delta$ ) from the configuration space. An example of the geometric braid and the trivial geometric braid which represents a unit element in $B_{N}$ are shown in Fig. 1. Since $\pi_{1}\left(R^{2}\right)=e$ and $R^{2}$ is simply connected, then

$$
B_{N}=\Sigma_{N}\left(R^{2}\right)
$$

and $B_{N}$ is generated by an elementary operation $\sigma_{i}$ of an interchange of two neighbouring particles. The geometric braids which represent $\sigma_{i}$ and $\sigma_{i}^{-1}$ are shown in Fig. 2. It is adopted compact that the string with label $i$ passes the string with
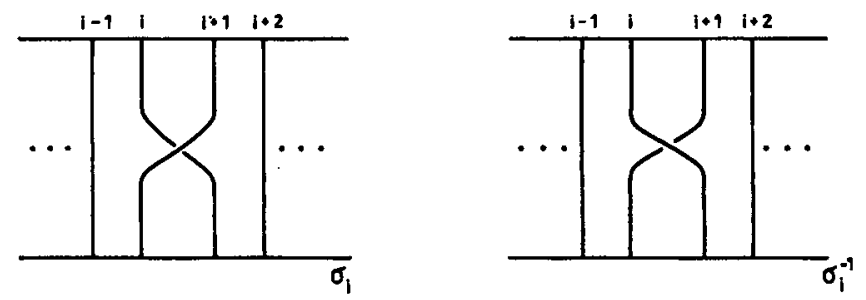

Fig. 2. Geometric braids corresponding to elementary operations of interchanging of two neighbouring particles, $\sigma_{i}$ and $\sigma_{i}^{-1}$.

label $i+1$ in front of it during the action of $\sigma_{i}$ and back of it during the action of $\sigma_{i}^{-1}$. It is easy to see that the geometric braid which represents $\sigma_{i} \cdot \sigma_{i}^{-1}$ is trivial. The relations which define generators $\sigma_{i}$ have the following form (cf. [10]):

$$
\sigma_{i} \cdot \sigma_{i+1} \cdot \sigma_{i}=\sigma_{i+1} \cdot \sigma_{i} \cdot \sigma_{i+1}
$$

where $1 \leq i \leq N-2$ and

$$
\sigma_{i} \cdot \sigma_{j}=\sigma_{j} \cdot \sigma_{i}
$$

where $1 \leq i, j \leq N-1,|i-j| \geq 2$. The geometric representations of these relations are shown in Fig. 3. It is very important that $\sigma_{i}^{2} \neq e$ (cf. Fig. 4. - though the points on both initial and final lines have the same order, the strings are hooked and cannot be disentangled without crossing). 


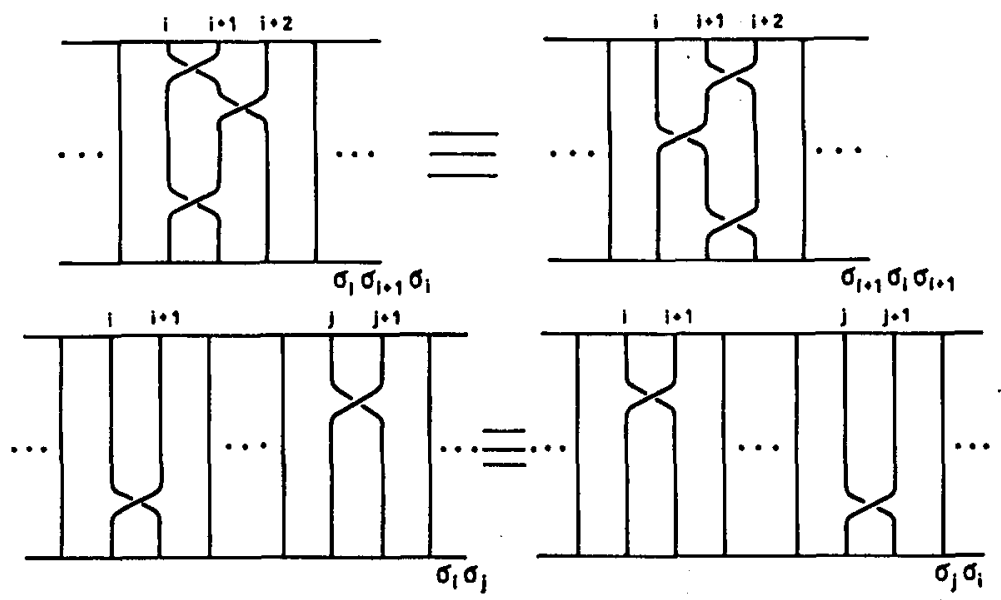

Fig. 3. Graphical illustration of the relation defining the generators $\sigma_{i}$ for the braid group $B_{N}$.

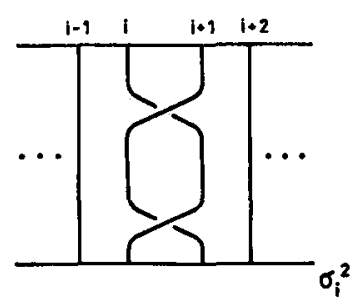

Fig. 4. Geometric braid for the element $\sigma_{i}^{2}$ for the braid group $B_{N}$.

Since $\pi_{1}\left(F_{N}\left(R^{2}\right)\right) \subset \Sigma_{N}\left(R^{2}\right)$ then generators $l \in \mathcal{L}$, which generate $\pi_{1}\left(F_{N}\left(R^{2}\right)\right)$, can be expressed by $\sigma_{i}$ as follows:

$$
l_{i j}=\sigma_{j-1} \cdot \sigma_{j-2} \cdot \ldots \sigma_{i+1} \cdot \sigma_{i}^{2} \cdot \sigma_{i+1}^{-1} \cdot \ldots \cdot \sigma_{j-2}^{-1} \cdot \sigma_{j-1}^{-1},
$$

where $1 \leq i<j \leq N-1$. An element of $\pi_{1}\left(F_{N}\left(R^{2}\right)\right)$ and the generator $l_{i j}$ are shown in Fig. 5. Let us consider the quotient group $B_{N} / \pi_{1}\left(F_{N}\left(R^{2}\right)\right)$. The quotient structure is connect with some homomorphism with a kernel being the normal subgroup of $\pi_{1}\left(F_{N}\left(R^{2}\right)\right)$. Hence, any string in the geometric braids cannot be hooked. This means that $\sigma_{i}^{2}=e$ and every element of such a group can be labelled by elements of a permutation group (the element which changes an initial order of particles to a final one). It can be written

$$
B_{N} / \pi_{1}\left(F_{N}\left(R^{2}\right)\right)=S_{N} .
$$

It turns out that the quotient structure $B_{N} / \pi_{1}\left(F_{N}\left(R^{2}\right)\right)$ is a group generated by the generators $\sigma_{i}$ satysfying relations (3) and (4) suplemented by another one

$$
\sigma_{i}^{2}=e \text {, }
$$

where $1 \leq i \leq N-1$. 


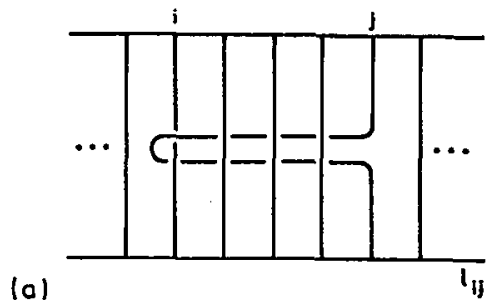

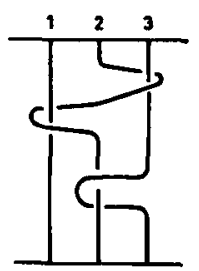

(b)

Fig. 5. Graphical illustration of some elements of the unpermuted braid group $\pi_{1}\left(F_{N}\left(R^{2}\right)\right)$ : a) the generator $\left.l_{i j}, \mathrm{~b}\right)$ an element $\pi_{1}\left(F_{3}\left(R^{2}\right)\right)$.

\section{Braid group for the sphere $S^{2}$}

The next example of the braid group for a two-dimensional manifold is $\pi_{1}\left(Q_{N}\left(S^{2}\right)\right)$. Since $\pi_{1}\left(S^{2}\right)=e$ then

$$
\pi_{1}\left(Q_{N}\left(S^{2}\right)\right)=\Sigma_{N}\left(S^{2}\right)
$$

and $\pi_{1}\left(Q_{N}\left(S^{2}\right)\right)$ is generated by elementary operations $\sigma_{i}$ of interchange neighbouring particles. A sphere is locally isomorphic with the Euclidean plane and hence relations (3) and (4), which define generators for $B_{N}$, are also satisfied for $S^{2}$. The global properties are, however, different and for that reason an additional relation appears. It is connected with the fact that each closed path in $Q_{N}\left(S^{2}\right)$ could be understood in two different ways. For example the path which describes a movement of one particle around all others particles could be regarded as a zero path. This double meaning of every closed paths provides additional relation (cf. [10])

$$
\sigma_{1} \cdot \sigma_{2} \cdot \ldots \cdot \sigma_{n-2} \cdot \sigma_{n-1}^{2} \cdot \sigma_{n-2} \cdot \ldots \cdot \sigma_{2} \cdot \sigma_{1}=e .
$$

Note that the relation (5) which represents generators $l_{i j}$ in terms of $\sigma_{i}$ is true for a sphere. Hence, similarly as in the last example, factoring $\pi_{1}\left(Q_{N}\left(S^{2}\right)\right)$ by $\pi_{1}\left(F_{N}\left(S^{2}\right)\right)$ is equivalent to adding the condition (7) to relations defining generators of $\pi_{1}\left(Q_{N}\left(S^{2}\right)\right)$ (for $S^{2}:(3),(4)$ and (8)) and also in this case

$$
\pi_{1}\left(Q_{N}\left(S^{2}\right)\right) / \pi_{1}\left(F_{N}\left(S^{2}\right)\right)=S_{N} \text {. }
$$

\section{Braid group for the torus $T$}

Another example is an $N$-particle system on the torus. In this case the situation is much more complicated since the first homotopy group for the torus is nontrivial. There are noncontractable loops even for a single particle and it must affect the first homotopy group for a many-particle configuration space. For that reason $\pi_{1}\left(Q_{N}(T)\right) \neq \Sigma_{N}(T)$. All generators of $\pi_{1}\left(Q_{N}(T)\right)$ fall into two separate sets (cf. [11]) and the generators of $\pi_{1}\left(F_{N}(T)\right)$ cannot be expressed in terms of elementary operations of interchange of neighbouring particles. The relations which define the generators $\sigma_{i}$ for the group $\Sigma_{N}(T)$ are exactly the same as in the case of the Euclidean plane (Eqs. (3) and (4)). The unpermuted braid group has two kinds of generators $\tau_{i}$ and $\rho_{i}$. Each of them transfers the particle $i$ along one of two fundamental noncontractable loops without moving any other particles as it 


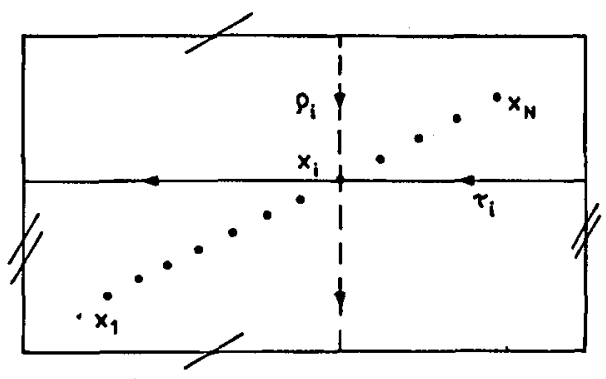

Fig. 6. The action of generators $\tau_{i}$ and $\rho_{i}$ of the unpermuted braid group $\pi_{1}\left(F_{N}(T)\right)$.

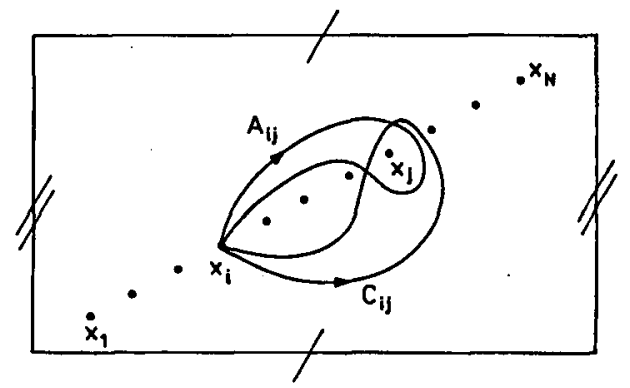

Fig. 7. The action of generators $A_{i j}$ and $C_{i j}$ of the unpermuted braid group $\pi_{1}\left(F_{N}(T)\right)$. is shown in Fig. 6. It is very convenient to introduce new generators $A_{i j}$ and $C_{i j}$ (cf. [11, 12]). Their actions are presented in Fig. 7. These additional generators can be expressed in terms of generators $\tau_{i}$ and $\rho_{i}$ as follows:

$$
\begin{aligned}
& A_{i j}=\rho_{i}^{-1} \cdot \tau_{i} \cdot \rho_{i} \cdot \tau_{i}^{-1}, \\
& C_{i j}=\tau_{i}^{-1} \cdot \rho_{i} \cdot \tau_{i} \cdot \rho_{i}^{-1},
\end{aligned}
$$

where $1 \leq i<j \leq n$. The relations which define generators of $\pi_{1}\left(F_{N}(T)\right)$ attain the following form:

$$
\begin{aligned}
& \tau_{k} \cdot A_{l m}=A_{l m} \cdot \tau_{k}, \quad \rho_{k} \cdot A_{l m}=A_{l m} \cdot \rho_{k}, \\
& \tau_{i} \cdot \tau_{j}=\tau_{j} \cdot \tau_{i}, \quad \rho_{i} \cdot \rho_{j}=\rho_{j} \cdot \rho_{i}, \\
& C_{i j}=\left(\tau_{i} \cdot \tau_{j}\right) \cdot A_{i j}^{-1} \cdot\left(\tau_{j}^{-1} \cdot \tau_{i}^{-1}\right), \\
& A_{i j}=\left(\rho_{i} \cdot \rho_{j}\right) \cdot C_{i j}^{-1} \cdot\left(\rho_{j}^{-1} \cdot \rho_{i}^{-1}\right), \\
& C_{i, j}=\left(A_{j-1, j}^{-1} \cdot \ldots \cdot A_{i+1, j}^{-1}\right) \cdot A_{i, j}^{-1} \cdot\left(A_{i+1, j} \cdot \ldots \cdot A_{j-1, j}\right), \\
& \tau_{1} \cdot \rho_{1} \cdot \tau_{1}^{-1} \cdot \rho_{1}^{-1}=A_{1, n} \cdot A_{1, n-1} \cdot \ldots \cdot A_{1,3} \cdot A_{1,2},
\end{aligned}
$$

where $1 \leq k<l<m \leq n$ and $1 \leq i<j \leq n$. There are also some additional relations which mix the generators of $\Sigma_{N}(T)$ and the generators of $\pi_{1}\left(F_{N}(T)\right.$ )

$$
\tau_{i+1}=\sigma_{i}^{-1} \cdot \tau_{i} \cdot \sigma_{i}^{-1}, \quad \rho_{i+1}=\sigma_{i} \cdot \rho_{i} \cdot \sigma_{i}
$$




$$
\begin{aligned}
& \tau_{i} \cdot \sigma_{j}=\sigma_{j} \cdot \tau_{i}, \quad \rho_{i} \cdot \sigma_{j}=\sigma_{j} \cdot \rho_{i}, \\
& \sigma_{i}^{2}=A_{i, i+1},
\end{aligned}
$$

where $1 \leq i \leq n-1$ and $2 \leq j \leq n-1$.

Note that the quotient group $\pi_{1}\left(Q_{N}(T)\right) / \tau_{1}\left(F_{N}(T)\right)$ can be obtained by including the condition

$$
\tau_{i}=\rho_{i}=e \text {. }
$$

Hence, $\pi_{1}\left(Q_{N}(T)\right) / \pi_{1}\left(F_{N}(T)\right)$ is generated by $\sigma_{i}$ which must satisfy relations (3) and (4) and additionally $\sigma_{i}^{2}=e$ (by virtue of Eqs. (10) and (20)). From the above it follows that

$$
\pi_{1}\left(Q_{N}(T)\right) / \pi_{1}\left(F_{N}(T)\right)=S_{N}
$$

\section{Braid group for the three-dimensional Euclidean space $R^{3}$}

Three-dimensional systems are most common in physics. In this section we prove that the braid group formalism leads in three dimensions to very well-known results, i.e. to Fermi-Dirac and Bose-Einstein statistics. Note that $\pi_{1}\left(R^{3}\right)=e$ and hence

$$
\pi_{1}\left(Q_{N}\left(R^{3}\right)\right)=\Sigma_{N}\left(R^{3}\right)
$$

and $\pi_{1}\left(Q_{N}\left(R^{3}\right)\right)$ is generated by $\sigma_{i}$. In the three-dimensional space singular points do not create topological defects and strings of geometric braids cannot hook themselves. Since each closed path in $F_{N}\left(R^{3}\right)$ is homotopic with the trivial loop then

$$
\pi_{1}\left(F_{N}\left(R^{3}\right)\right)=e .
$$

Thus every braids which have the same initial and final points belong to the same class of homotopy and refer to the same element of $\pi_{1}\left(Q_{N}\left(R^{3}\right)\right)$. This means that the elements of $\pi_{1}\left(Q_{N}\left(R^{3}\right)\right)$ are labelled by elements of $S_{N}$, and, in fact

$$
\pi_{1}\left(Q_{N}\left(R^{3}\right)\right)=S_{N} \text {. }
$$

In the case of $R^{3}$ the relations similar to (6), (9) and (21) can be also obtained $\pi_{1}\left(Q_{N}\left(R^{3}\right)\right) / \pi_{1}\left(F_{N}\left(R^{3}\right)\right)=S_{N} / e=S_{N}$.

The relations which define generators $\sigma_{1}$ for the permutation group $S_{N}$ are the same as for $B_{N}$ (Eqs. (3) and (4)) with one additional relation

$$
\sigma_{i}^{2}=e \text {, }
$$

for $1 \leq i \leq n-1$. The most important difference between $R^{2}$ and $R^{3}$ is that in $R^{2}$ there are two different reasons for noncontractability of loops: hooking of strings and interchanging of particle order, but in $R^{3}$ only the second one remains.

If one considers the braid group for a simply connected manifold with $\operatorname{dim} M>3$ one obtains a similar situation as for $R^{3}$, and

$$
\pi_{1}\left(Q_{N}(M)\right)=S_{N},
$$

since for all manifolds $\operatorname{dim} M \geq 3, \pi_{1}(M)=e$. Even though $\operatorname{dim} M \geq 3$ and $\pi_{1}(M) \neq e, \pi_{1}\left(Q_{N}(M)\right)$ becomes different than $S_{N}$ but still $\Sigma_{N}(M)=S_{N}$ in all those cases. Thus topology of $M$ in higher-dimensional spaces $M$ can influence $\pi_{1}\left(Q_{N}(M)\right)$ by changing $\pi_{1}\left(F_{N}(M)\right)$ but it cannot change $\Sigma_{N}(M)$. 


\section{Braid groups for the line $R^{1}$ and the circle $S^{1}$}

For manifilds with $\operatorname{dim} M \geq 2$ the idea of the braid group is natural. In one dimension the similar construction could have, however, a quite different structure. For example, if $M$ is the infinite Euclidean line $R^{1}$ then the braid group of such a system is very simple. The space $F_{N}\left(R^{1}\right)=R^{N} \backslash \Delta$ resolves itself into separate subspaces $C_{i}$ and each of them is a maximal simply connected subspace. They are labelled by elements of the permutation group $S_{N}$. This means that if the initial point of a path is in $C_{i}$ then the final point of this path is also in $C_{i}$. The physical meaning of this fact is very simple. If the coincidence of particle coordinates is excluded then the order of particles on the line cannot be changed. Each subspace $C_{i}$ contains all points of the configuration space which describe the same order of particles. From the above it follows that

$$
\pi_{1}\left(Q_{N}\left(R^{1}\right)\right)=\pi_{1}\left(F_{N}\left(R^{1}\right)\right)=e .
$$

There is a much more complex situation when $M=S^{1}$. In this case particles can change their order but only in a very limited way. For example, let three particles can move around a circle from the initial order $1,2,3, \ldots, n$ to the final one $4,5, \ldots, n, 1,2,3$. We see that only cyclic permutations of the initial order are possible. It is easy to note, that the operations from the sets $\mathcal{L}$ and $\mathcal{P}$ cannot be generators of $\pi_{1}\left(Q_{N}\left(S^{1}\right)\right)$ and the group $\Sigma_{N}\left(S^{1}\right)$ does not exist at all (particles cannot interchange in the sense of the group $\Sigma_{N}$; it is not true for all one-dimensional manifolds). The group $\pi_{1}\left(Q_{N}\left(S^{1}\right)\right)$ is generated by the sole generator $\delta$. The path related to $\delta$ is a movement of the first particle around the circle to the end position without moving any other particles (more exactly, particles with indices $i \neq 1$ take the positions with indices $i-1)$. The generator $\delta$ is of infinite order because even if $\delta$ acts $n$ times and initial and final orders are the same $\delta^{n}$ is not the neutral element of $\pi_{1}\left(Q_{N}\left(S^{1}\right)\right)$ because the closed path related to $\delta^{n}$ is noncontractactable (the path winds up on the circle). From the above it follows that

$$
\pi_{1}\left(Q_{N}\left(S^{1}\right)\right)=Z \text {. }
$$

The unpermuted braid group $\pi_{1}\left(F_{N}\left(S^{1}\right)\right)$ is also generated by the single generator $\nu$. It is referred to the sole revolution of the whole system around the circle. The generator $\nu$ can be expressed by the generator $\delta$

$$
\nu=\delta^{N} \text {. }
$$

From the above it follows that

$$
\pi_{1}\left(F_{N}\left(S^{1}\right)\right)=Z \text {. }
$$

Thus $\pi_{1}\left(Q_{N}\left(S^{1}\right)\right)$ and $\pi_{1}\left(F_{N}\left(S^{1}\right)\right)$ are referred to the same abstract group but they are defined by two different generators. It is also easy to show that

$$
\pi_{1}\left(Q_{N}\left(S^{1}\right)\right) / \pi_{1}\left(F_{N}\left(S^{1}\right)\right)=Z_{N}
$$

where $Z_{N}$ is the cyclic group of order $N$ (it is generated by $\delta$ with the relation $\left.\delta^{N}=e\right)$. 


\section{Exact sequences for the braid groups}

Some relations found in the previous sections can be easy obtained in a more formal way. As it was seen the configuration space $F_{N}(M)$ is the covering space for $Q_{N}(M)$. For this fibration a long exact sequence of the homotopy group can be written (cf. [13])

$$
\begin{aligned}
& \rightarrow \pi_{n}\left(\left(F_{N}(M)\right)_{x}, y\right) \rightarrow \pi_{n}\left(F_{N}(M), y\right) \rightarrow \pi_{n}\left(Q_{N}(M), x\right) \rightarrow \pi_{n-1}\left(\left(F_{N}(M)\right)_{x}, y\right) \\
& \rightarrow \pi_{n-1}\left(F_{N}(M), y\right) \rightarrow \pi_{n-1}\left(Q_{N}(M), x\right) \rightarrow \ldots \rightarrow \pi_{1}\left(\left(F_{N}(M)\right)_{x}, y\right) \\
& \rightarrow \pi_{1}\left(F_{N}(M), y\right) \rightarrow \pi_{1}\left(Q_{N}(M), x\right) \rightarrow \pi_{0}\left(\left(F_{N}(M)\right)_{x}, y\right) \rightarrow \pi_{0}\left(F_{N}(M), y\right), \quad(30)
\end{aligned}
$$

where $x \in Q_{N}(M), y \in\left(F_{N}(M)\right)_{x}, \pi_{n}$ is the $n$-th homotopy group and the arrow indicates homorphism. Because each fibre $\left(F_{N}(M)\right)_{x}$ is discrete then

$$
\pi_{1}\left(\left(F_{N}(M)\right)_{x}, y\right)=e
$$

for each $x \in Q_{N}(M)$ and $y \in\left(F_{N}(M)\right)_{x}$. Moreover, if $M$ is arcwise connected and $\operatorname{dim} M \geq 2$ then also $F_{N}(M)$ and $Q_{N}(M)$ are arcwise connected. Then base points can be omitted ( $x$ and $y$ points in following formulae):

$$
\begin{aligned}
& \pi_{1}\left(F_{N}(M), y\right)=\pi_{1}\left(F_{N}(M)\right) \\
& \pi_{1}\left(Q_{N}(M), x\right)=\pi_{1}\left(Q_{N}(M)\right)
\end{aligned}
$$

and also

$$
\pi_{0}\left(F_{N}(M), y\right)=e \text {. }
$$

Therefore, five last terms of the long exact sequence of homotopy groups give the following short exact sequence in the form

$$
e \rightarrow \pi_{1}\left(F_{N}(M)\right) \rightarrow \pi_{1}\left(Q_{N}(M)\right) \rightarrow \pi_{0}\left(\left(F_{N}(M)\right)_{x}, y\right) \rightarrow e .
$$

$\left(F_{N}(M)\right)_{x}$ is a discrete space of the order of $N$ ! and their points are labelled by elements of the group $S_{N}$. It is easy to see that

$$
\pi_{0}\left(\left(F_{N}(M)\right)_{x}, y\right)=S_{N} \text {. }
$$

Finally, the short exact sequence attains the form

$$
e \rightarrow \pi_{1}\left(F_{N}(M)\right) \stackrel{\alpha}{\rightarrow} \pi_{1}\left(Q_{N}(M)\right) \stackrel{\beta}{\rightarrow} S_{N} \rightarrow e
$$

and every generators of $\pi_{1}\left(F_{N}(M)\right)$ are collected in the kernel of epimorphism $\beta$. Since $\pi_{1}\left(F_{N}(M)\right)$ is a normal subgroup of the braid group $\pi_{1}\left(Q_{N}(M)\right)$ then

$$
\pi_{1}\left(Q_{N}(M)\right) / \pi_{1}\left(F_{N}(M)\right)=S_{N},
$$

for all arcwise connected manifolds $M, \operatorname{dim} M \geq 2$. This relation is similar to those obtained for $R^{2}, S^{2}, T, R^{3}$.

It is obvious that for all arcwise connected manifolds the quotient group $\pi\left(Q_{N}(M)\right) / \pi_{1}\left(F_{N}(M)\right)$ is a group which generates all final configurations from arbitrary initial configuration of particles (for $\operatorname{dim} M \geq 2$ all final configurations are attainable). For the line $R^{1}, \pi_{1}\left(Q_{N}\left(R^{1}\right)\right) / \pi_{1}\left(F_{N}\left(R^{1}\right)\right)=e$ and any initial configuration cannot be changed. For the circle $S^{1}, \pi_{1}\left(Q_{N}\left(S^{1}\right)\right) / \pi_{1}\left(F_{N}\left(S^{1}\right)\right)=S_{N}^{c}$ and only the cyclic permutations of the initial order are allowed. 


\section{Quantization of the many-particle system}

The standard procedure of the quantization of a physical system with the configuration space $Q_{N}(M)$ is to construct a fixed in time quantum-mechanical state as a function $\psi$ from $Q_{N}(M)$ into complex numbers $C$ (cf. [14, 15]). In general, $\psi$ can be multivalued: $\psi_{m}, m=1,2, \ldots, L$. The quantum theory with one-component wave function $\psi$ is called the scalar quantum theory. There is only a restriction that if an argument of $\psi$ is taken along closed paths in $Q_{N}(M)$ the state vector $\psi$ must transform itself according to a unitary representation of a braid group $\pi_{1}\left(Q_{N}(M)\right)$ ( $L$-dimensional representation for $L$-dimensional quantum theory). For distinct irreducible unitary representations (IUR) of the braid group (even of the same dimension) there are distinct quantum theories for the same classical system. Since the braid group describes the interchange of particles then it must be connected with quantum statistics. Not the whole braid group describes replacement of particles but rather its subgroup $\Sigma_{N}$ (cf. also [14]). For example, $\pi_{1}\left(Q_{1}(M)\right)$ could be very complicated for some $M$ but it is a one-particle system and there is no base to introduce an idea of quantum statistics (in this case $\pi_{1}\left(Q_{1}(M)\right)=\pi_{1}\left(F_{1}(M)\right)$ and the unpermuted braid group does not describe exchanging of particles). Some elements of $\pi_{1}\left(F_{N}(M)\right)$ could be connected with replacement of particles but each of these elements must also be an element of $\Sigma_{N}$. The quantum statistics cannot be described by IUR of $\Sigma_{N}(M)$ because it is not the full symmetry group of the system - one has to take into account restrictions of IUR due to $\pi_{1}\left(Q_{N}(M)\right)$. It is especially important when $\pi_{1}(M) \neq e$ and the set of generators of $\pi_{1}\left(Q_{N}(M)\right)$ falls into sets $\mathcal{L}$ and $\mathcal{P}$. The relations which mix generators from $\mathcal{L}$ and $\mathcal{P}$ restrict possible IUR of $\Sigma_{N}(M)$.

For the scalar statistics connected with one-dimensional IUR it is very useful to compute the first homology group of $Q_{N}(M)$ or equivalently the abelised braid group (cf. [14]). In this case it is sufficient to give the value of real parameters $\Theta_{i}$ for all generators and the representation attains the form of $\Phi_{\Theta_{i}}\left(\sigma_{i}\right)=\mathrm{e}^{\mathrm{i} \Theta_{i}}$. From the relations (3) and (4) which appear in every two-, three- and higher-dimensional examples of manifolds (as relation defining generators of $\Sigma_{N}(M)$ ) it follows that

$$
\Theta_{1}=\Theta_{2}=\ldots=\Theta_{n-1}=\Theta
$$

Thus there is one real parameter which labels IUR of $\Sigma_{N}(M)$. For particular $M$ there are, however, some additional relations restricting possible values of $\Theta$.

For the Euclidean plane $\Sigma_{N}\left(R^{2}\right)=\pi_{1}\left(Q_{N}\left(R^{2}\right)\right)$ and

$$
\left[\pi_{1}\left(Q_{N}\left(R^{2}\right)\right)\right]_{\mathrm{ab}}=Z
$$

and $\Theta$ is of any value from $[0, \pi)$. There is no additional relation imposed on possible values of $\Theta$. leads to

For the sphere $\Sigma_{N}\left(S^{2}\right)=\pi_{1}\left(Q_{N}\left(S^{2}\right)\right)$ but another relation appears (8). It

$$
\left[\pi_{1}\left(Q_{N}\left(S^{2}\right)\right)\right]_{\mathrm{ab}}=Z_{2 N-2} .
$$

This restricts the value of $\Theta$ and

$$
\Theta=\frac{k \cdot \pi}{N-1}
$$


where $k=1,2, \ldots, 2 N-3$. One can see that if $\Theta$ is fixed then not every number of particles on the sphere is allowed.

The case of the torus is much more complex [16]

$$
\left[\pi_{1}\left(Q_{N}(T)\right)\right]_{\mathrm{ab}}=H_{1}(T) \oplus Z_{2} \text {, }
$$

where $H_{1}(T)$ is the first homology group for the torus. The image of $\Sigma_{N}(T)$ under the abelisation map is $Z_{2}$. Hence, the restriction of one-dimensional IUR of $\pi_{1}\left(Q_{N}(T)\right)$ group to $\Sigma_{N}(T)$ can be numbered by $\Theta=0$ and $\pi$ which refers to bosons and fermions, respectively. This result one can obtain from the relations (10) and (20) (they lead to the condition $\Phi\left(\sigma_{i}^{2}\right)=1$ ). It is seen that there exists the difference between IUR of $\Sigma_{N}(T)$ group and the restrictions of IUR of $\pi_{1}\left(Q_{N}(T)\right)$ group to $\Sigma_{N}(T)$ (for $\Sigma_{N}(T)$ all $\Theta$ from $[0, \pi)$ are allowed). This indicates that quantum statistics is connected with boundary conditions - the torus is the rectangular plaquette with the periodic boundary condition imposed. Note that the relation (34) is true for each closed two-dimensional manifold $M \neq S^{2}[16]$ and hence there is no possibility of existing nonstandard and one-dimensional statistics for such manifolds.

For three- and higher-dimensional simply connected manifolds $M$, $\pi_{1}\left(Q_{N}(M)\right)=S_{N}$ and

$\left[S_{N}\right]_{\mathrm{ab}}=Z_{2}$.

In all these cases only $\Theta=0$ and $\Theta=\pi$ are allowed. This leads to symmetric or antisymmetric wave functions, respectively and there are no other possibilities (for scalar quantum theories). Even if $\pi_{1}(M) \neq e$ it does not change this situation. It is obvious because if $\operatorname{dim} M>1$ and $M$ is arcwise connected then $S_{N}$ is the poorest braid group and any additional relation cannot restrict its representation. Hence, for these manifolds we deal only with two scalar statistics: boson- and fermion-like statistics.

For the line $R^{1}$ there is no reason for introducing quantum statistics in the sense of the braid group formalism, but for the circle $S^{1}$ it is not so clear. Even though $\Sigma_{N}\left(S^{1}\right)$ does not exist at all, the particles can change their order. This problem seems to be still open. One could say that every element for $\pi_{1}\left(Q_{N}(M)\right)$ can be expressed as decomposition into pure interchanging of particles and pure unpermuted braiding. For the circle it is, however, impossible.

Supported by the project No. 202369101 of the Committee for the Scientific Research.

\section{References}

[1] L. Schulman, Phys. Rev. 176, 1558 (1968).

[2] M.G. Laidlaw, C.M. De Witt, Phys. Rev. D 3, 1375 (1971).

[3] J.M. Leinaas, J. Myrheim, Nuovo Cimento B 37, 1 (1977).

[4] B.C. Tsui, H.L. Stormer, Phys. Rev. Lett. 48, 1559 (1982).

[5] J.G. Bednorz, K.A. Muller, Z. Phys. B, Condens. Mater 64, 189 (1986). 
[6] F. Wilczek, Phys. Rev. Lett. 48, 1144 (1982).

[7] F. Wilczek, Phys. Rev. Lett. 49, 957 (1982).

[8] R. Laughlin, Science 242, 525 (1988).

[9] E. Spanier, Algebraic Topology, Springer Verlag, Berlin 1966.

[10] J.S. Birman, Braids, Links and Mapping Class Groups, Princeton U.P., New York 1974.

[11] J.S. Birman, Comm. Pure Appl. Math. 22, 41 (1969).

[12] T. Einarsson, Phys. Rev. Lett. 64, 1995 (1990).

[13] R. Duda, Wprowadzenie do topologii, PWN, Warszawa 1986 (in Polish).

[14] T.D. Imbo, C.S. Imbo, E.C.G. Sudarshan, Phys. Lett. B 234, 103 (1990).

[15] E.C.G. Sudarshan, Phys. Lett. B 213, 471 (1988).

[16] G.P. Scott, Proc. Camb. Phil. Soc. 68, 6705 (1970). 